\title{
UNIFORM ATTRACTORS FOR A NON-AUTONOMOUS SEMILINEAR HEAT EQUATION WITH MEMORY
}

\author{
BY \\ CLAUDIO GIORGI (Dipartimento di Matematica, Università di Brescia, I-25133 Brescia, Italy), \\ VITTORINO PATA (Dipartimento di Matematica, Università di Brescia, I-25133 Brescia, Italy),
} AND

ALFREDO MARZOCCHI (Dipartimento di Matematica, Università Cattolica del S.Cuore, I-25121 Brescia, Italy)

\begin{abstract}
In this paper we investigate the asymptotic behavior, as time tends to infinity, of the solutions of a non-autonomous integro-partial differential equation describing the heat flow in a rigid heat conductor with memory. Existence and uniqueness of solutions is provided. Moreover, under proper assumptions on the heat flux memory kernel and on the magnitude of nonlinearity, the existence of uniform absorbing sets and of a global uniform attractor is achieved. In the case of quasiperiodic dependence of time of the external heat supply, the above attractor is shown to have finite Hausdorff dimension.
\end{abstract}

0. Introduction. Let $\Omega \subset \mathbb{R}^{3}$ be a fixed bounded domain occupied by a rigid, isotropic, homogeneous heat conductor with linear memory. We consider the following integro-partial differential equation, which is derived in the framework of the wellestablished theory of heat flow with memory due to Coleman and Gurtin [8]:

$$
\begin{aligned}
& c_{0} \frac{\partial}{\partial t} \theta-k_{0} \Delta \theta-\int_{-\infty}^{t} k(t-s) \Delta \theta(s) d s+g(\theta)=h \quad \text { on } \Omega \times(\tau,+\infty), \\
& \theta(x, t)=0, \quad x \in \partial \Omega, t>\tau, \\
& \theta(x, \tau)=\theta_{0}(x), \quad x \in \Omega,
\end{aligned}
$$

Received June, 1998.

2000 Mathematics Subject Classification. Primary 35B40, 35K05, 45K05, 47H20.

Key words and phrases. Heat equation, heat flux memory kernel, asymptotic behavior, uniform absorbing set, uniform attractor, translation compact functions.

This work was done under the auspices of GNAFA and GNFM - CNR and was partially supported by the Italian MURST through the projects "Mathematical Models for Materials Science" and "Problems and Methods in the Theory of Hyperbolic Equations".

E-mail address: giorgi@bsing.ing.unibs.it

E-mail address: pata@bsing.ing.unibs.it

E-mail address: marz012@dmf.bs.unicatt.it 
where $\theta: \Omega \times \mathbb{R} \rightarrow \mathbb{R}$ is the temperature variation field relative to the equilibrium reference value, $k: \mathbb{R}^{+} \rightarrow \mathbb{R}$ is the heat flux memory kernel, and the constants $c_{0}$ and $k_{0}$ denote the specific heat and the instantaneous conductivity, respectively. The function $h: \Omega \times[\tau,+\infty) \rightarrow \mathbb{R}$ is a time-dependent external heat source, whereas $g: \mathbb{R} \rightarrow \mathbb{R}$ is a nonlinear heat supply.

System (0.1) was studied in [14], assuming a time-independent external heat source $h$ and a polynomial nonlinearity $g$. Along the line of the procedure suggested by Dafermos in his pioneer work [11], we introduce the new variables

$$
\theta^{t}(x, s)=\theta(x, t-s), \quad s \geq 0
$$

and

$$
\eta^{t}(x, s)=\int_{0}^{s} \theta^{t}(x, y) d y=\int_{t-s}^{t} \theta(x, y) d y, \quad s \geq 0 .
$$

Assuming $k(\infty)=0$, performing a change of variable, and setting

$$
\mu(s)=-k^{\prime}(s)
$$

formal integration by parts transforms the above system (0.1) into

$$
\begin{aligned}
& c_{0} \frac{\partial}{\partial t} \theta-k_{0} \Delta \theta-\int_{0}^{\infty} \mu(s) \Delta \eta^{t}(s) d s+g(\theta)=h \quad \text { on } \Omega \times(\tau,+\infty), \\
& \frac{\partial}{\partial t} \eta^{t}(x, s)=\theta(x, t)-\frac{\partial}{\partial s} \eta^{t}(x, s), \quad x \in \Omega, t>\tau, s>0 \\
& \theta(x, t)=0, \quad x \in \partial \Omega, t>\tau, \\
& \theta(x, \tau)=\theta_{0}(x), \quad x \in \Omega \\
& \eta^{\tau}(x, s)=\eta_{0}(x, s), \quad x \in \Omega, s>0
\end{aligned}
$$

where the term

$$
\eta^{\tau}(x, s)=\int_{0}^{s} \theta^{\tau}(x, y) d y=\int_{\tau-s}^{\tau} \theta(x, y) d y, \quad s \geq 0
$$

is the prescribed initial integrated past history of $\theta(x, t)$, which does not depend on $\theta_{0}(x)$, and is assumed to vanish on $\partial \Omega$, as well as $\theta(x, t)$. As a consequence, it follows that

$$
\eta^{t}(x, s)=0, \quad x \in \partial \Omega, t>\tau, s>0 .
$$

Indeed, the above assertion is obvious if $\tau \leq t-s$, and if $\tau>t-s$ we can write

$$
\eta^{t}(x, s)=\eta_{0}(x, \tau+s-t)+\int_{\tau}^{t} \theta(x, y) d y
$$

In the sequel we agree to denote by $\partial_{t}$ or more simply by $t_{t}$ the derivation with respect to $t$, and denote by a prime the derivation with respect to $s$.

The constitutive quantities $c_{0}, k_{0}$, and $\mu$ are required to verify the following set of hypotheses:

(h1) $c_{0}>0, \quad k_{0}>0$

(h2) $\mu \in C^{1}\left(\mathbb{R}^{+}\right) \cap L^{1}\left(\mathbb{R}^{+}\right), \quad \mu(s) \geq 0, \mu^{\prime}(s) \leq 0, \forall s \in \mathbb{R}^{+}$;

(h3) $\mu^{\prime}(s)+\delta \mu(s) \leq 0 \quad \forall s \in \mathbb{R}^{+}$and some $\delta>0$. 
Notice that (h3) implies the exponential decay of $\mu(s)$. Nevertheless, it allows $\mu(s)$ to have a singularity at $s=0$, whose order is less than 1 , since $\mu(s)$ is a nonnegative $L^{1}$-function.

Now, taking for simplicity $c_{0}=1$, and denoting $z(t)=\left(\theta(t), \eta^{t}\right), z_{0}=\left(\theta_{0}, \eta_{0}\right)$, and setting

$$
L z=\left(k_{0} \Delta \theta+\int_{0}^{\infty} \mu(s) \Delta \eta(s) d s, \theta-\eta^{\prime}\right)
$$

and

$$
G(z)=(h-g(\theta), 0),
$$

problem (0.2) assumes the compact form

$$
\begin{aligned}
z_{t} & =L z+G(z), \\
z(x, t) & =0, \quad x \in \partial \Omega, t>\tau, \\
z(x, \tau) & =z_{0} .
\end{aligned}
$$

We recall that existence, uniqueness, and stability of the linear problem corresponding to $(0.1)$ (i.e., with $g \equiv 0$ ) have been investigated by several authors (see, e.g., [13, 15, 18, 19]). Related results, which include phase transition phenomena, are in $[3,9]$. On the other hand, when no memory effect occurs, long-time behavior of semilinear parabolic problems like $(0.1)$ with $k \equiv 0$ have been widely studied, both for autonomous and nonautonomous equations (see, e.g., $[5,6,7,22]$ ). However, the main aim of this paper is to study the role played by the memory term as time tends to infinity. Results concerning asymptotic behavior of solutions for semilinear problems in the presence of nontrivial terms of convolution type, involving the principal part of the differential operator, can be found in $[1,12,14,16]$.

In this paper, due to the time-dependence of $h$, the evolutive system (0.3) of differential equations is non-autonomous. Therefore, in order to study its asymptotic behavior, we have to introduce the notion of process, which is a generalization of the semigroup of operators on a Hilbert space.

DeFinition 0.1. A two-parameter family $\{U(t, \tau)\}_{t \geq \tau, \tau \in \mathbb{R}}$ of operators on a Hilbert space $\mathcal{H}$ is said to be a process if the following hold:

(i) $U(\tau, \tau)$ is the identity map on $\mathcal{H}$ for any $\tau \in \mathbb{R}$;

(ii) $U(t, s) U(s, \tau)=U(t, \tau)$ for any $t \geq s \geq \tau$;

(iii) $U(t, \tau) x \rightarrow x$ as $t \downarrow \tau$ for any $x \in \mathcal{H}$ and any $\tau \in \mathbb{R}$.

In Theorem 2.1 and Theorem 2.2 below we show that, given $z_{0}$ in a suitable Hilbert space $\mathcal{H}$, under proper conditions on $g$ and $h$, there exists a unique solution $z(t)$ in $\mathcal{H}$ to problem $(0.3)$, which continuously depends on $z_{0}$. Thus, we can define the family of processes $U_{h}(t, \tau)$, depending on the functional parameter $h$, and acting on $\mathcal{H}$, as

$$
U_{h}(t, \tau) z_{0}=z(t)
$$

where $z(t)$ is the solution at time $t$ of $(0.3)$ with initial data $z_{0}$ given at time $\tau$. The parameter $h$ is usually called the symbol of the process. Notice that if $h$ is time-independent, the process $U_{h}(t, \tau)$ reduces to a semigroup by setting

$$
S_{h}(t)=U_{h}(t, 0)
$$


The study of the long-time behavior of the family of processes $U_{h}(t, \tau)$ will be carried out in Sec. 3 and Sec. 4, where, using the techniques of $[5,6]$, we prove the existence of absorbing sets in $\mathcal{H}$ and in a smaller space $\mathcal{V}$, and of a global attractor. In all cases, the objects are uniform since $h$ is allowed to move in a suitable functional space. Finally, in Sec. 5 we show that for a particular choice of the symbol space (namely for an external heat supply with a quasiperiodic dependence on time) the Hausdorff dimension of the uniform attractor is finite.

1. Functional setting and notation. Let $\Omega \subset \mathbb{R}^{3}$ be a bounded domain with Lipschitz boundary. With the usual notation, we introduce the spaces $L^{p}, H^{k}$, and $H_{0}^{k}$ acting on $\Omega$. Let $\langle\cdot, \cdot\rangle$ and $\|\cdot\|$ denote the $L^{2}$-inner product and the $L^{2}$-norm, respectively, and let $\|\cdot\|_{p}$ denote the $L^{p}$-norm. With abuse of notation, we use $\langle\cdot, \cdot\rangle$ to denote also the duality between $L^{p}$ and its dual space $L^{q}$. We recall the Poincaré inequality

$$
\lambda_{0}\|v\|^{2} \leq\|\nabla v\|^{2} \quad \forall v \in H_{0}^{1}
$$

and

$$
\gamma_{0}\|\nabla v\|^{2} \leq\|\Delta v\|^{2} \quad \forall v \in H^{2} \cap H_{0}^{1}
$$

for some $\lambda_{0}, \gamma_{0}>0$, where (1.2) is obtained from (1.1) and the Young inequality (see Lemma 1.2 below). By force of (1.1)-(1.2), the inner products on $H_{0}^{1}$ and $H^{2} \cap H_{0}^{1}$ can be defined in the following manner:

$$
\langle u, v\rangle_{H_{0}^{1}}=\langle\nabla u, \nabla v\rangle
$$

and

$$
\langle u, v\rangle_{H^{2} \cap H_{0}^{1}}=\langle\Delta u, \Delta v\rangle .
$$

In view of $(\mathrm{h} 2)$, let $L_{\mu}^{2}\left(\mathbb{R}^{+}, L^{2}\right)$ be the Hilbert space of $L^{2}$-valued functions on $\mathbb{R}^{+}$, endowed with the inner product

$$
\langle\varphi, \psi\rangle_{\mu}=\int_{0}^{\infty} \mu(s)\langle\varphi(s), \psi(s)\rangle d s .
$$

Similarly on $L_{\mu}^{2}\left(\mathbb{R}^{+}, H_{0}^{1}\right)$ and $L_{\mu}^{2}\left(\mathbb{R}^{+}, H^{2} \cap H_{0}^{1}\right)$, respectively, we have the inner products

$$
\langle\varphi, \psi\rangle_{L_{\mu}^{2}\left(\mathbb{R}^{+}, H_{0}^{1}\right)}=\langle\nabla \varphi, \nabla \psi\rangle_{\mu}
$$

and

$$
\langle\varphi, \psi\rangle_{L_{\mu}^{2}\left(\mathbb{B}^{+}, H^{2} \cap H_{0}^{1}\right)}=\langle\Delta \varphi, \Delta \psi\rangle_{\mu} .
$$

Finally, we introduce the Hilbert spaces

$$
\mathcal{H}=L^{2} \times L_{\mu}^{2}\left(\mathbb{R}^{+}, H_{0}^{1}\right)
$$

and

$$
\mathcal{V}=H_{0}^{1} \times L_{\mu}^{2}\left(\mathbb{R}^{+}, H^{2} \cap H_{0}^{1}\right),
$$

which are, respectively, endowed with the inner products

$$
\left\langle w_{1}, w_{2}\right\rangle_{\mathcal{H}}=\left\langle\psi_{1}, \psi_{2}\right\rangle+\left\langle\Delta \varphi_{1}, \Delta \varphi_{2}\right\rangle_{\mu}
$$


and

$$
\left\langle w_{1}, w_{2}\right\rangle_{\mathcal{V}}=\left\langle\nabla \psi_{1}, \nabla \psi_{2}\right\rangle+\left\langle\Delta \varphi_{1}, \Delta \varphi_{2}\right\rangle_{\mu}
$$

where $w_{i}=\left(\psi_{i}, \varphi_{i}\right) \in \mathcal{H}$ or $\mathcal{V}$ for $i=1,2$.

We will also consider spaces of functions defined on a (possibly infinite) interval $I$ with values in a Banach space $X$ such as $C(I, X), L^{p}(I, X)$, and $H^{k}(I, X)$, with the usual norms.

To describe the asymptotic behavior of the solutions of our system we need to introduce the space $\mathcal{T}_{b}^{p}(\mathbb{R}, X)$ of $L_{\text {loc }}^{P}$-translation bounded functions with values in a Banach space $X$, namely

$$
\mathcal{T}_{b}^{p}(\mathbb{R}, X)=\left\{f \in L_{\mathrm{loc}}^{p}(\mathbb{R}, X):\|f\|_{\mathcal{T}_{b}^{p}(\mathbb{R}, X)}=\sup _{\xi \in \mathbb{R}}\left(\int_{\xi}^{\xi+1}\|f(y)\|_{X}^{p} d y\right)^{1 / p}<\infty\right\} .
$$

In an analogous manner, given $\tau \in \mathbb{R}$, we define the space $\mathcal{T}_{b}^{p}([\tau,+\infty), X)$.

Definition 1.1. A function $f \in L_{\text {loc }}^{p}(\mathbb{R}, X)$ is said to be translation compact in $L_{\text {loc }}^{p}(\mathbb{R}, X)$, and write $f \in \mathcal{T}_{c}^{p}(\mathbb{R}, X)$, if the hull of $f$, defined as

$$
H(f)=\overline{\{f(\cdot+r)\}_{r \in \mathbb{R}}} L_{\mathrm{loc}}^{p}(\mathbb{R}, X),
$$

is compact in $L_{\text {loc }}^{p}(\mathbb{R}, X)$.

The reader is referred to [7] for a more detailed presentation of the subject. Here we just highlight that $\mathcal{T}_{c}^{p}(\mathbb{R}, X) \subset \mathcal{T}_{b}^{p}(\mathbb{R}, X)$. Moreover,

$$
\|\varphi\|_{\mathcal{T}_{b}^{p}(\mathbb{R}, X)} \leq\|f\|_{\mathcal{T}_{b}^{p}(\mathbb{R}, X)} \quad \forall \varphi \in H(f) .
$$

We also remark that the class $\mathcal{T}_{c}^{p}(\mathbb{R}, X)$ is quite general. For example, it contains $L^{q}(\mathbb{R}, X)$ for all $q \geq p$, the constant $X$-valued functions, and the class of almost periodic functions (see [2]).

We now recall some technical results which will be needed in the course of the investigation.

Lemma 1.2 (Young inequality). Let $a, b \geq 0$ be given. Then for every $\varepsilon>0$, and for every $1<p, q<\infty$ such that $\frac{1}{p}+\frac{1}{q}=1$, the inequality

$$
a b \leq \varepsilon a^{p}+K(\varepsilon, p, q) b^{q}
$$

holds with

$$
K(\varepsilon, p, q)=\frac{1}{q}(\varepsilon p)^{-q / p} .
$$

LEmma 1.3 (Gagliardo-Nirenberg). Let $2<p \leq 6$. Then there exists $c>0$ such that the inequality

$$
\|u\|_{p} \leq c\|\nabla u\|^{\nu}\|u\|^{1-\nu}
$$

holds for all $u \in H_{0}^{1}$, with

$$
\nu=\frac{3}{2}\left[\frac{p-2}{p}\right]
$$


Here and in the sequel, for $\tau \in \mathbb{R}$, we denote $\mathbb{R}_{\tau}=[\tau,+\infty)$. The following Gronwalltype lemma is a modification of Lemme A.5 in [4] (see [20] for a detailed proof).

LEMmA 1.4. Let $\phi$ be a nonnegative, absolutely continuous function on $\mathbb{R}_{\tau}, \tau \in \mathbb{R}$, that satisfies for some $\varepsilon>0$ and $0 \leq \sigma<1$ the differential inequality

$$
\frac{d}{d t} \phi(t)+\varepsilon \phi(t) \leq \Lambda+m_{1}(t) \phi(t)^{\sigma}+m_{2}(t) \quad \text { a.e. } t \in \mathbb{R}_{\tau}
$$

where $\Lambda \geq 0$, and $m_{1}$ and $m_{2}$ are nonnegative locally summable functions on $\mathbb{R}_{\tau}$. Then

$$
\begin{aligned}
\phi(t) \leq & \frac{1}{1-\sigma}\left[\phi(\tau) e^{-\varepsilon(t-\tau)}+\frac{\Lambda}{\varepsilon}\right]+\left[\int_{\tau}^{t} m_{1}(y) e^{-\varepsilon(1-\sigma)(t-y)} d y\right]^{1 /(1-\sigma)} \\
& +\frac{1}{1-\sigma} \int_{\tau}^{t} m_{2}(y) e^{-\varepsilon(t-y)} d y
\end{aligned}
$$

for any $t \in \mathbb{R}_{\tau}$.

The easy proof of the next result is left to the reader.

Lemma 1.5. Let $m \in \mathcal{T}_{b}^{1}\left(\mathbb{R}_{\tau}, \mathbb{R}^{+}\right)$for some $\tau \in \mathbb{R}$. Then, for every $\varepsilon>0$,

$$
\int_{\tau}^{t} m(y) e^{-\varepsilon(t-y)} d y \leq C(\varepsilon)\|m\|_{\mathcal{T}_{b}^{1}\left(\mathbb{R}_{\tau}, \mathbb{R}^{+}\right)}
$$

where

$$
C(\varepsilon)=\frac{e^{\varepsilon}}{1-e^{-\varepsilon}} .
$$

We conclude the section with a lemma which will be needed in the last part of the paper (for the proof see [22], pp. 300-303).

Lemma 1.6. There exists a positive constant $\kappa$ such that, for any given $m$ vectors $\left\{\varphi_{1}, \ldots, \varphi_{m}\right\}$ in $H_{0}^{1}$ that are orthonormal in $L^{2}$, it follows that

$$
\sum_{j=1}^{m}\left\|\nabla \varphi_{j}\right\|^{2} \geq \kappa m^{5 / 3}
$$

2. Existence and Uniqueness. In this section we establish existence and uniqueness results for problem (0.3). Unlike in [14], where the nonlinearity is assumed to be an odd-degree polynomial of any order, here we are interested in considering more general nonlinear terms. Of course we have to pay the price of requiring additional properties. Here below is a list of conditions on $g$ which will be used throughout the paper.

Conditions on the nonlinear term $g$. Let $g \in C(\mathbb{R})$, and assume that there exist nonnegative constants $c_{j}, j=1,2,3,4,5$, and $\beta>0$ such that

$$
\begin{array}{ll}
\text { (g1) } & |g(u)| \leq c_{1}\left(1+|u|^{\beta}\right) \\
\text { (g2) } & u \cdot g(u) \geq-c_{2}+c_{3}|u|^{\beta+1} \\
\text { (g3) } & |g(u)-g(v)| \leq c_{4}|u-v|\left(1+|u|^{\gamma}+|v|^{\gamma}\right) \quad \text { with } \gamma=\max \{\beta-1,0\} \\
\text { (g4) } & g \in C^{1}(\mathbb{R}) \quad \text { and } \quad g^{\prime}(u) \geq-c_{5} .
\end{array}
$$


Clearly, if the nonlinearity is weak, the above conditions can be relaxed. For instance, if $\beta \leq 1$ then $c_{3}=0$, and if $\beta<1$ then (g2) is not needed at all.

Theorem 2.1. Assume (h1)-(h2), and let (g1)-(g2) hold for some $\beta>0$. For any initial time $\tau \in \mathbb{R}$, given

$$
h \in L_{\mathrm{loc}}^{1}\left(\mathbb{R}_{\tau}, L^{2}\right)+L_{\mathrm{loc}}^{2}\left(\mathbb{R}_{\tau}, H^{-1}\right) \quad \text { and } \quad z_{0}=\left(\theta_{0}, \eta_{0}\right) \in \mathcal{H}
$$

there exists a function $z=(\theta, \eta)$, with

$$
\begin{aligned}
& \theta \in L^{\infty}\left([\tau, T], L^{2}\right) \cap L^{2}\left([\tau, T], H_{0}^{1}\right) \cap L^{\beta+1}\left([\tau, T], L^{\beta+1}\right) \quad \forall T>\tau, \\
& \eta \in L^{\infty}\left([\tau, T], L_{\mu}^{2}\left(\mathbb{R}^{+}, H_{0}^{1}\right)\right) \quad \forall T>\tau,
\end{aligned}
$$

such that

$$
z_{t}=L z+G(z)
$$

in the weak sense, and

$$
\left.z\right|_{t=\tau}=z_{0}
$$

Furthermore,

$$
z \in C([0, T], \mathcal{H}) \quad \forall T>\tau
$$

Proof of the above result recasts exactly the Faedo-Galerkin scheme used in [14]. The only difference here depends on the presence of a time-dependent heat supply $h \in$ $L_{\text {loc }}^{1}\left(\mathbb{R}_{\tau}, L^{2}\right)+L_{\text {loc }}^{2}\left(\mathbb{R}_{\tau}, H^{-1}\right)$, which can be easily handled making use of the Gronwall lemma.

We just recall two relations from [14] which will be needed later. The first is obtained by taking the inner product in $\mathcal{H}$ of $(0.3)_{1}$ and $z=(\theta, \eta)$, applying the divergence theorem, performing an integration by parts, and using (h2), whereas the second one is obtained by integrating the first one from $\tau$ to $T$, along with proper estimates:

$$
\begin{gathered}
\frac{d}{d t}\|z\|_{\mathcal{H}}^{2}+2 k_{0}\|\nabla \theta\|^{2}-\int_{0}^{\infty} \mu^{\prime}(s)\|\nabla \eta(s)\|^{2} d s=-2\langle g(\theta), \theta\rangle+2\langle h, \theta\rangle, \\
\int_{\tau}^{T}\|\theta(y)\|_{\beta+1}^{\beta+1} d y \leq C\left(\left\|\theta_{0}\right\|^{2}+T\right)
\end{gathered}
$$

for some $C>0$ independent of $T$.

In order to obtain uniqueness results for $(0.3)$, further restrictions on $g$ are needed. We shall provide two uniqueness results under different hypotheses.

THEOREM 2.2. In the hypotheses of Theorem 2.1, assume that either (g3) with $\beta \leq \frac{7}{3}$ or $(\mathrm{g} 4)$ hold. Then the solution $z(t)$ to $(0.3)$ is unique, and the mapping

$$
z_{0} \mapsto z(t) \in C(\mathcal{H}, \mathcal{H}) \quad \forall t \in[\tau, T]
$$

Proof. Suppose that $z_{1}=\left(\theta_{1}, \eta_{1}\right)$ and $z_{2}=\left(\theta_{2}, \eta_{2}\right)$ are two solutions of $(0.3)$ with initial data $z_{10}$ and $z_{20}$, respectively, and set $\tilde{z}=(\tilde{\theta}, \tilde{\eta})=z_{1}-z_{2}$ and $\tilde{z}_{0}=z_{10}-z_{20}$. 
Taking the difference of $(0.3)_{1}$ with $z_{1}$ and $z_{2}$ in place of $z$, and taking the product by $\tilde{z}$ in $\mathcal{H}$, we get (repeating the argument leading to $(2.1)$ )

$$
\frac{d}{d t}\|\tilde{z}\|_{\mathcal{H}}^{2}+2 k_{0}\|\nabla \tilde{\theta}\|^{2} \leq-2\left\langle g\left(\theta_{1}\right)-g\left(\theta_{2}\right), \tilde{\theta}\right\rangle
$$

where we used (h2) to delete the integral term.

Suppose first that (g3) holds with $\beta \leq \frac{7}{3}$. Since

$$
2\left|\left\langle g\left(\theta_{1}\right)-g\left(\theta_{2}\right), \tilde{\theta}\right\rangle\right| \leq 2 c_{4} \int_{\Omega}|\tilde{\theta}|^{2}\left[1+\left|\theta_{1}\right|^{\gamma}+\left|\theta_{2}\right|^{\gamma}\right] d x,
$$

applying the generalized Hölder inequality with $p, q \geq 1$ such that

$$
\frac{1}{6}+\frac{1}{p}+\frac{1}{q}=1
$$

and by virtue of the continuity of the embedding $H_{0}^{1} \hookrightarrow L^{6}$,

$$
\begin{aligned}
2\left|\left\langle g\left(\theta_{1}\right)-g\left(\theta_{2}\right), \tilde{\theta}\right\rangle\right| & \leq 2 c_{4}\|\tilde{\theta}\|_{6}\|\tilde{\theta}\|_{p}\left\|1+\left|\theta_{1}\right|^{\gamma}+\left|\theta_{2}\right|^{\gamma}\right\|_{q} \\
& \leq c_{6}\|\nabla \tilde{\theta}\|\|\tilde{\theta}\|_{p}\left\|1+\left|\theta_{1}\right|^{\gamma}+\left|\theta_{2}\right|^{\gamma}\right\|_{q}
\end{aligned}
$$

for some $c_{6}>0$. We now consider two cases. If $\gamma \leq 1$, then choose $p=2$ and $q=3$ in (2.4), and define

$$
m_{\gamma}(t)=\frac{c_{6}^{2}}{8 k_{0}}\left\|1+\left|\theta_{1}(t)\right|^{\gamma}+\left|\theta_{2}\right|^{\gamma}\right\|_{3}^{2} .
$$

Since $3 \gamma \leq \gamma+2$, and $\theta_{1}, \theta_{2} \in L^{\beta+1}\left([\tau, T], L^{\beta+1}\right)$, it is clear that $m_{\gamma} \in L^{1}([\tau, T])$, and by force of (2.2), $\left\|m_{\gamma}\right\|_{L^{1}([\tau, T])}$ remains bounded as $z_{10}$ and $z_{20}$ run in a bounded set. By the Young inequality,

$$
2\left|\left\langle g\left(\theta_{1}\right)-g\left(\theta_{2}\right), \tilde{\theta}\right\rangle\right| \leq 2 k_{0}\|\nabla \tilde{\theta}\|^{2}+m_{\gamma}\|\tilde{\theta}\|^{2} .
$$

If $1<\gamma \leq \frac{4}{3}$, let

$$
p=\frac{6 \gamma+12}{10-\gamma} \quad \text { and } \quad q=\frac{\gamma+2}{\gamma}
$$

in (2.4). From Lemma 1.3,

$$
\|\tilde{\theta}\|_{p} \leq c\|\nabla \tilde{\theta}\|^{\nu}\|\tilde{\theta}\|^{1-\nu}
$$

with

$$
\nu=2\left[\frac{\gamma-1}{\gamma+2}\right]
$$

Therefore, (2.4) enhances to

$$
2\left|\left\langle g\left(\theta_{1}\right)-g\left(\theta_{2}\right), \tilde{\theta}\right\rangle\right| \leq c c_{6}\|\nabla \tilde{\theta}\|^{1+\nu}\|\tilde{\theta}\|^{1-\nu}\left\|1+\left|\theta_{1}\right|^{\gamma}+\left|\theta_{2}\right|^{\gamma}\right\|_{q} .
$$

Define

$$
m_{\gamma}(t)=\left(c c_{6}\right)^{2 /(1-\nu)} K\left(2 k_{0}, \frac{2}{1+\nu}, \frac{2}{1-\nu}\right)\left\|1+\left|\theta_{1}\right|^{\gamma}+\left|\theta_{2}\right|^{\gamma}\right\|_{q}^{2 /(1-\nu)}
$$

with $K$ as in (1.3). Notice that $\gamma q=\gamma+2$, and $\frac{2 \gamma}{1-\nu} \leq \gamma+2$ for $\gamma \leq \frac{4}{3}$. Since $\theta_{1}, \theta_{2} \in L^{\gamma+2}\left([\tau, T], L^{\gamma+2}\right)$, we conclude that $m_{\gamma} \in L^{1}([\tau, T])$, and again from (2.2), 
$\left\|m_{\gamma}\right\|_{L^{1}([\tau, T])}$ remains bounded as $z_{10}$ and $z_{20}$ run in a bounded set. Applying Lemma 1.2 to (2.7) we get that inequality (2.6) holds also in this case. Thus (2.3) turns into

$$
\frac{d}{d t}\|\tilde{z}\|_{\mathcal{H}}^{2} \leq m_{\gamma}\|\tilde{\theta}\|^{2} \leq m_{\gamma}\|\tilde{z}\|_{\mathcal{H}}^{2}
$$

and the Gronwall lemma then yields

$$
\|\tilde{z}(t)\|_{\mathcal{H}}^{2} \leq\left\|\tilde{z}_{0}\right\|_{\mathcal{H}}^{2} \exp \left[\int_{\tau}^{t} m_{\gamma}(y) d y\right],
$$

which implies the result.

Assume then (g4), and notice that

$$
g\left(\theta_{1}\right)-g\left(\theta_{2}\right)=\tilde{\theta} \int_{0}^{1} g^{\prime}\left(\lambda \theta_{1}+(1-\lambda) \theta_{2}\right) d \lambda .
$$

Therefore,

$$
\begin{aligned}
-2\left\langle g\left(\theta_{1}\right)-g\left(\theta_{2}\right), \tilde{\theta}\right\rangle & =-2 \int_{\Omega}|\tilde{\theta}|^{2}\left[\int_{0}^{1} g^{\prime}\left(\lambda \theta_{1}+(1-\lambda) \theta_{2}\right) d \lambda\right] d x \\
& \leq 2 c_{5}\|\tilde{\theta}\|^{2}
\end{aligned}
$$

and (2.3) becomes

$$
\frac{d}{d t}\|\tilde{z}\|_{\mathcal{H}}^{2} \leq 2 c_{5}\|\tilde{\theta}\|^{2} \leq 2 c_{5}\|\tilde{z}\|_{\mathcal{H}}^{2} .
$$

Using again the Gronwall lemma we obtain

$$
\|\tilde{z}(t)\|_{\mathcal{H}}^{2} \leq\left\|\tilde{z}_{0}\right\|_{\mathcal{H}}^{2} e^{2 c_{5}(t-\tau)},
$$

which concludes the proof.

3. Existence of Uniform Absorbing Sets in $\mathcal{H}$ and in $\mathcal{V}$. Let $\mathcal{E}$ be the Hilbert space into which move all orbits of problem (0.3), namely

$$
U_{h}(t, \tau): \mathcal{E} \rightarrow \mathcal{E}, \quad \mathcal{E}=\mathcal{H} \text { or } \mathcal{E}=\mathcal{V},
$$

with $U_{h}(t, \tau)$ given by $(0.4)$. The aim of this section is to prove in either case the existence of a bounded absorbing set, which is uniform as $h$ runs into a given functional set, typically a complete metric space. Such a set is sometimes called the symbol space.

In the sequel $F$ will always denote a symbol space. We also agree to call $B_{\mathcal{E}}(0, R)$ the open ball in $\mathcal{E}$ with center 0 and radius $R>0$.

Definition 3.1. A set $\mathcal{B}_{0} \subset \mathcal{E}$ is said to be uniformly absorbing (with respect to $h \in F$ ) for the family $\left\{U_{h}(t, \tau), h \in F\right\}$ if for any bounded set $\mathcal{B} \subset \mathcal{E}$ there exists $t^{*}=t^{*}(\mathcal{B})$ such that

$$
\bigcup_{h \in F} U_{h}(t, \tau) \mathcal{B} \subset \mathcal{B}_{0} \quad \forall t \geq \tau+t^{*}, \forall \tau \in \mathbb{R} .
$$

Theorem 3.2. Assume (h1)-(h3), and let (g1)-(g2), and either (g3) with $\beta \leq \frac{7}{3}$, or (g4) hold. Let

$$
F \subset \mathcal{T}_{b}^{1}\left(\mathbb{R}, L^{2}\right)+\mathcal{T}_{b}^{2}\left(\mathbb{R}, H^{-1}\right)
$$


be a bounded set. Then there exists a bounded, uniformly absorbing set in $\mathcal{H}$ for the family $\left\{U_{h}(\tau, t), h \in F\right\}$.

Proof. Let

$$
\begin{aligned}
\Phi & =\sup _{h \in F}\|h\|_{\mathcal{T}_{b}^{1}\left(\mathbb{R} . L^{2}\right)+\mathcal{T}_{b}^{2}\left(\mathbb{R} . H^{-1}\right)} \\
& =\sup _{h \in F}\left[\inf _{h=h_{1}+h_{2}}\left\{\left\|h_{1}\right\|_{\mathcal{T}_{b}^{1}\left(\mathbb{R}, L^{2}\right)}+\left\|h_{2}\right\|_{\mathcal{T}_{b}^{2}\left(\mathbb{R}, H^{-1}\right)}\right\}\right]
\end{aligned}
$$

and let $h=h_{1}+h_{2}$ be a decomposition of $h$. By force of (g2) and Young's inequality we get

$$
-2\langle g(\theta), \theta\rangle+2\langle h, \theta\rangle \leq 2 c_{2}|\Omega|-2 c_{3}\|\theta\|_{\beta+1}^{\beta+1}+2\left\|h_{1}\right\|\|\theta\|+\frac{k_{0}}{2}\|\nabla \theta\|^{2}+\frac{2}{k_{0}}\left\|h_{2}\right\|_{H^{-1}}^{2} .
$$

Thus, using (h3) and (1.1), and denoting

$$
\varepsilon_{0}=\min \left\{\lambda_{0} k_{0}, \delta\right\} \quad \text { and } \quad \Lambda=2 \max \left\{c_{2}|\Omega|, 1, \frac{1}{k_{0}}\right\},
$$

Eq. (2.1) turns into

$$
\frac{d}{d t}\|z\|_{\mathcal{H}}^{2}+\varepsilon_{0}\|z\|_{\mathcal{H}}^{2}+k_{0}\|\nabla \theta\|^{2}+2 c_{3}\|\theta\|_{\beta+1}^{\beta+1} \leq \Lambda\left[1+\left\|h_{1}\right\|\|z\|_{\mathcal{H}}+\left\|h_{2}\right\|_{\mathcal{H}^{-1}}^{2}\right] .
$$

Therefore, setting

$$
\Psi=\Psi\left(\varepsilon_{0}, \Lambda, \Phi\right)=\frac{2 \Lambda}{\varepsilon_{0}}+\Lambda^{2} \Phi^{2} C^{2}\left(\frac{\varepsilon_{0}}{2}\right)+2 \Lambda \Phi^{2} C\left(\varepsilon_{0}\right)
$$

with $C$ as in (1.4), Lemma 1.4 with $\sigma=\frac{1}{2}$ and Lemma 1.5 entail

$$
\|z(t)\|_{\mathcal{H}}^{2} \leq 2\left\|z\left(t_{0}\right)\right\|_{\mathcal{H}}^{2} e^{-\varepsilon_{0}\left(t-t_{0}\right)}+\Psi \quad \forall t \geq t_{0}
$$

for any given $t_{0}>\tau$. In particular,

$$
\|z(t)\|_{\mathcal{H}}^{2} \leq 2\left\|z_{0}\right\|_{\mathcal{H}}^{2} e^{-\varepsilon_{0}(t-\tau)}+\Psi \quad \forall t \geq \tau,
$$

from which it follows at once that every ball $B_{\mathcal{H}}(0, \rho)$, with radius $\rho>\sqrt{\Psi}$, is a uniformly absorbing set in $\mathcal{H}$ as $h \in F$.

Observe that, in the above proof, condition (g3) with $\beta \leq \frac{7}{3}$ or (g4) are used only to formulate the result in terms of process (and therefore uniqueness of solution is required). If we relax (g3) and (g4), the very same uniform bound holds for a (not necessarily unique) solution of $(0.3)$.

In order to get an absorbing set in $\mathcal{V}$, we have to strengthen the hypothesis on the symbol space.

Theorem 3.3. Assume (h1)-(h3), and let (g1)-(g2), and either (g3) with $\beta<\frac{7}{3}$, or (g4) holds. Let

$$
F \subset \mathcal{T}_{b}^{2}\left(\mathbb{R}, L^{2}\right)
$$

be a bounded set. Then there exists a bounded, uniformly absorbing set in $\mathcal{V}$ for the family $\left\{U_{h}(\tau, t), h \in F\right\}$. 
Proof. Let

$$
\Phi=\sup _{h \in F}\|h\|_{\mathcal{T}_{b}^{2}\left(\mathbb{R}, L^{2}\right)} .
$$

Observe that (3.1)-(3.3) still hold (with $h_{1}=h$ and $\left.h_{2} \equiv 0\right)$. Hence, integration of (3.1) on the interval $[t, t+1]$, with $t \geq \tau$, and Young's inequality lead to the estimate

$$
\begin{aligned}
\int_{t}^{t+1} & {\left[k_{0}\|\nabla \theta(y)\|^{2}+2 c_{3}\|\theta(y)\|_{\beta+1}^{\beta+1}\right] d y } \\
& \leq\|z(t)\|_{\mathcal{H}}^{2}+\Lambda+\int_{t}^{t+1} \Lambda\|h(y)\|\|z(y)\|_{\mathcal{H}} d y \\
& \leq(2+\Lambda)\left\|z\left(t_{0}\right)\right\|_{\mathcal{H}}^{2}+\Psi+\Lambda+\frac{1}{2} \Lambda \Phi^{2}+\frac{1}{2} \Lambda \Psi \quad \forall t \geq t_{0}
\end{aligned}
$$

for any given $t_{0} \geq \tau$. To achieve uniform estimates involving the existence of a bounded uniformly absorbing set in $\mathcal{V}$, we multiply $(0.2)_{1}$ by $-\Delta \theta$ with respect to the inner product of $L^{2}$, and the Laplacian of $(0.2)_{2}$ by $\Delta \eta$ with respect to the inner product of $L_{\mu}^{2}\left(\mathbb{R}^{+}, L^{2}\right)$. Adding the two terms, and performing an integration by parts, we obtain

$$
\frac{d}{d t}\|z\|_{\mathcal{V}}^{2}+2 k_{0}\|\Delta \theta\|^{2}-\int_{0}^{\infty} \mu^{\prime}(s)\|\Delta \eta(s)\|^{2} d s=2\langle g(\theta)-h, \Delta \theta\rangle .
$$

Young's inequality entails

$$
2|\langle h, \Delta \theta\rangle| \leq \frac{k_{0}}{2}\|\Delta \theta\|^{2}+\frac{2}{k_{0}}\|h\|^{2} .
$$

Concerning the term $2\langle g(\theta), \Delta \theta\rangle$, assume first that (g3) holds with $\beta<\frac{7}{3}$. Young's inequality and $(\mathrm{g} 1)$ then give

$$
\begin{aligned}
2\langle g(\theta), \Delta \theta\rangle & \leq \frac{k_{0}}{2}\|\Delta \theta\|^{2}+\frac{2 c_{1}^{2}}{k_{0}}\left\|1+|\theta|^{\beta}\right\|^{2} \\
& \leq \frac{k_{0}}{2}\|\Delta \theta\|^{2}+c_{7}+c_{8}\|\theta\|_{2 \gamma+2}^{2 \gamma+2}
\end{aligned}
$$

for some $c_{7}, c_{8}>0$. From Lemma 1.3 (recall that $\gamma=\max \{\beta-1,0\}$ ),

$$
\|\theta\|_{2 \gamma+2}^{2 \gamma+2} \leq c\|\nabla \theta\|^{3 \gamma}\|\theta\|^{2-\gamma} .
$$

Hence, introducing

$$
\sigma=\frac{1}{2} \max \{3 \gamma-2,0\}<1
$$

we get

$$
2\langle g(\theta), \Delta \theta\rangle \leq \frac{k_{0}}{2}\|\Delta \theta\|^{2}+c_{7}+c c_{8}\left(1+\|\theta\|^{2}\right)\left(1+\|\nabla \theta\|^{2}\right)\|\nabla \theta\|^{2 \sigma} .
$$

Consider next the case when (g4) holds. Since $\left.\theta\right|_{\partial \Omega}=0$, the continuity of $g$ and the Green formula yield

$$
\int_{\partial \Omega} g(\theta) \nabla \theta \cdot \mathbf{n} d \sigma=\int_{\partial \Omega} g(0) \nabla \theta \cdot \mathbf{n} d \sigma=\int_{\Omega} g(0) \Delta \theta d x
$$


where $\mathbf{n}$ is the outward pointing normal vector. Thus, using again the Green formula, by force of Young's inequality and (g4),

$$
\begin{aligned}
2\langle g(\theta), \Delta \theta\rangle & =2 \int_{\Omega} g(0) \Delta \theta d x-2 \int_{\Omega} g^{\prime}(\theta) \nabla \theta \cdot \nabla \theta d x \\
& \leq \frac{2}{k_{0}} g^{2}(0)|\Omega|+\frac{k_{0}}{2}\|\Delta \theta\|^{2}+2 c_{5}\|\nabla \theta\|^{2} .
\end{aligned}
$$

Clearly the above computations are justified in a Faedo-Galerkin scheme. Therefore in either case, setting

$$
\varphi_{1}=c c_{8}\left(1+\|\theta\|^{2}\right)\left(1+\|\nabla \theta\|^{2}\right)
$$

and

$$
\varphi_{2}=\frac{2}{k_{0}}\|h\|^{2}+c_{7}+\frac{2}{k_{0}} g^{2}(0)|\Omega|+2 c_{5}\|\nabla \theta\|^{2},
$$

from (3.7)-(3.9) we conclude that

$$
2\langle g(\theta)-h, \Delta \theta\rangle \leq \varphi_{1}\|\nabla \theta\|^{2 \sigma}+\varphi_{2}+k_{0}\|\Delta \theta\|^{2} .
$$

Finally, by virtue of (h3) and (1.2), and denoting

$$
\varepsilon_{1}=\min \left\{\gamma_{0} k_{0}, \delta\right\}
$$

we obtain from (3.6) the inequality

$$
\frac{d}{d t}\|z\|_{\mathcal{V}}^{2}+\varepsilon_{1}\|z\|_{\mathcal{V}}^{2} \leq \varphi_{1}\|z\|_{\mathcal{V}}^{2 \sigma}+\varphi_{2}
$$

From (3.3) and (3.5), there exist two positive constants $K_{1}$ and $K_{2}$ (depending on $\Phi$ ) such that

$$
\int_{t}^{t+1}\left[\varphi_{1}(y)+\varphi_{2}(y)\right] d y \leq K_{1}\left\|z\left(t_{0}\right)\right\|_{\mathcal{H}}^{4}+K_{2} \quad \forall t \geq t_{0}
$$

for any given $t_{0} \geq \tau$. Hence Lemma 1.4 and Lemma 1.5 lead to

$$
\begin{aligned}
\|z(t)\|_{\mathcal{V}}^{2} \leq & \frac{1}{1-\sigma}\left\|z\left(t_{0}\right)\right\|_{\mathcal{V}}^{2} e^{-\varepsilon_{1}(t-\tau)}+\left[C\left(\varepsilon_{1}(1-\sigma)\right)\left(K_{1}\left\|z\left(t_{0}\right)\right\|_{\mathcal{H}}^{4}+K_{2}\right)\right]^{1 /(1-\sigma)} \\
& +\frac{1}{1-\sigma} C\left(\varepsilon_{1}\right)\left(K_{1}\left\|z\left(t_{0}\right)\right\|_{\mathcal{H}}^{4}+K_{2}\right) \quad \forall t \geq t_{0},
\end{aligned}
$$

for any given $t_{0} \geq \tau$. Let now $z_{0} \in B(0, R)$ in $\mathcal{V}$. Recalling (1.1)-(1.2),

$$
\left\|z_{0}\right\|_{\mathcal{H}} \leq R_{1}=R \max \left\{\frac{1}{\lambda_{0}}, \frac{1}{\gamma_{0}}\right\}
$$

and (3.10) applied for $t_{0}=\tau$ yields

$$
\|z(t)\|_{\mathcal{V}}^{2} \leq \frac{1}{1-\sigma} R^{2}+\xi\left(R_{1}\right) \quad \forall t \geq \tau
$$

having defined the function

$$
\xi(r)=\left[C\left(\varepsilon_{1}(1-\sigma)\right)\left(K_{1} r^{4}+K_{2}\right)\right]^{1 /(1-\sigma)}+\frac{1}{1-\sigma} C\left(\varepsilon_{1}\right)\left(K_{1} r^{4}+K_{2}\right) .
$$


Notice that $\xi(r)$ is increasing in $r$. Set now, with reference to $(3.2), \rho>\sqrt{\Psi}$. According to Theorem 3.2 there exists $t_{R} \geq 0$ such that $\|z(t)\|_{\mathcal{H}} \leq \rho$ whenever $t \geq \tau+t_{R}$. Thus from (3.10)-(3.11) we get that

$$
\|z(t)\|_{\mathcal{V}}^{2} \leq \frac{R^{2}+\xi\left(R_{1}\right)(1-\sigma)}{(1-\sigma)^{2}} e^{-\varepsilon_{1}(t-\tau)}+\xi(\rho) \quad \forall t \geq \tau+t_{R} .
$$

Therefore, we get that every ball $B_{\mathcal{V}}\left(0, \rho^{\prime}\right)$, with $\rho^{\prime}>\sqrt{\xi(\sqrt{\Psi})}$, is a uniformly absorbing set in $\mathcal{V}$ as $h \in F$.

4. Existence of a Uniform Attractor. We begin recalling some definitions due to Haraux [17].

Definition 4.1. A set $\mathcal{K} \subset \mathcal{H}$ is said to be uniformly attracting for the family $\left\{U_{h}(t, \tau), h \in F\right\}$ if for any $\tau \in \mathbb{R}$ and any bounded set $\mathcal{B} \subset \mathcal{H}$,

$$
\lim _{t \rightarrow \infty}\left[\sup _{h \in F} \operatorname{dist}\left(U_{h}(t, \tau) \mathcal{B}, \mathcal{K}\right)\right]=0,
$$

where

$$
\operatorname{dist}\left(\mathcal{B}_{1}, \mathcal{B}_{2}\right)=\sup _{z_{1} \in \mathcal{B}_{1}} \inf _{z_{2} \in \mathcal{B}_{2}}\left\|z_{1}-z_{2}\right\|_{\mathcal{H}}
$$

denotes the semidistance of two sets $\mathcal{B}_{1}$ and $\mathcal{B}_{2}$ in $\mathcal{H}$. A family of processes that possesses a uniformly attracting compact set is said to be uniformly asymptotically compact.

Definition 4.2. A closed set $\mathcal{A} \subset \mathcal{H}$ is said to be a uniform attractor for the family $\left\{U_{h}(t, \tau), h \in F\right\}$ if it is at the same time uniformly attracting and contained in every closed uniformly attracting set.

The above minimality property replaces the invariance property that characterizes the attractors of semigroups. It is also clear from the definition that the uniform attractor of a family of processes is unique.

The fundamental results of Chepyzhov and Vishik (see $[5,6]$ ) that we are going to exploit read as follows.

THEOREM 4.3. Assume that $F$ is a compact metric space and that there exists a continuous semigroup $T(t)$ acting on it that satisfies the translation equality

$$
U_{h}(t+s, \tau+s)=U_{T(s) h}(t, \tau) \quad \forall h \in F .
$$

Assume also that $U_{h}(t, \tau)$ is continuous as a map $\mathcal{H} \times F \rightarrow \mathcal{H}$, for every $\tau \in \mathbb{R}$ and $t \geq \tau$. Then if the family $\left\{U_{h}(t, \tau), h \in F\right\}$ is uniformly asymptotically compact, it possesses a compact uniform attractor given by

$$
\mathcal{A}=\left\{\begin{array}{l}
z(0) \text { such that } z(t) \text { is any bounded complete } \\
\text { trajectory of } U_{h}(t, \tau) \text { for some } h \in \mathcal{A}(F)
\end{array}\right\}
$$

where $\mathcal{A}(F)$ is the attractor of the semigroup $T(t)$ on $F$.

The existence of $\mathcal{A}(F)$ is assured from the well-known theorems about the attractors of semigroups (see, e.g., [22]). 
THEOREM 4.4. The set $\widetilde{\mathcal{A}}=\mathcal{A} \times \mathcal{A}(F) \subset \mathcal{H} \times F$ is the attractor of the semigroup $S(t)$ acting on $\mathcal{H} \times F$ defined by

$$
S(t)\left(z_{0}, h\right)=\left(U_{h}(t, 0) z_{0}, T(t) h\right) .
$$

From the translation equality (4.2), it is immediate to verify that $S(t)$ is a semigroup. Using standard techniques, one could prove directly the existence of an attractor $\widetilde{\mathcal{A}}$ for $S(t)$. The peculiarity of Theorem 4.3 and Theorem 4.4 stands in the characterization of $\widetilde{\mathcal{A}}$ as $\mathcal{A} \times \mathcal{A}(F)$.

In view of the above results, we write the solution $z=(\theta, \eta)$ to $(0.3)$ as $z=z_{L}+z_{N}$, with $z_{L}=\left(\theta_{L}, \eta_{L}\right)$ and $z_{N}=\left(\theta_{N}, \eta_{N}\right)$, where $z_{L}$ solves the linearized homogeneous system, and $z_{N}$ is the solution of the nonlinear system with null initial data, namely,

$$
\begin{aligned}
\partial_{t} z_{L} & =L z_{L}, \\
\left.z_{L}\right|_{\partial \Omega} & =0, \\
z_{L}(0) & =z_{0},
\end{aligned}
$$

and

$$
\begin{aligned}
\partial_{t} z_{N} & =L z_{N}+G(z), \\
\left.z_{N}\right|_{\partial \Omega} & =0 \\
z_{N}(0) & =0 .
\end{aligned}
$$

It is apparent that the solution $z_{L}$ to (4.4) fulfills the uniform estimate (3.4) with $\Psi=0$, namely,

$$
\left\|z_{L}(t)\right\|_{\mathcal{H}}^{2} \leq 2\left\|z_{0}\right\|_{\mathcal{H}}^{2} e^{-\varepsilon_{0}(t-\tau)} \quad \forall t \geq \tau
$$

Since

$$
\left\|z_{N}(t)\right\|_{\mathcal{H}}^{2} \leq 2\|z(t)\|_{\mathcal{H}}^{2}+2\left\|z_{L}(t)\right\|_{\mathcal{H}}^{2},
$$

we also have

$$
\left\|z_{N}(t)\right\|_{\mathcal{H}}^{2} \leq 8\left\|z_{0}\right\|_{\mathcal{H}}^{2} e^{-\varepsilon_{0}(t-\tau)}+2 \Psi \quad \forall t \geq \tau .
$$

For further reference, we denote by $\eta_{N}^{t}\left(s ; \tau, z_{0}, h\right)$ the second component of the solution $z_{N}$ to (4.5) at time $t$ with initial time $\tau$ with $z(\tau)=z_{0}$ and symbol $h$. Observe that $\eta_{N}$ can be computed explicitly from (4.5) as follows:

$$
\eta_{N}^{t}(s)=\left\{\begin{array}{l}
\int_{0}^{s} \theta_{N}(t-y) d y \quad 0 \leq s \leq t-\tau, \\
\int_{0}^{t-\tau} \theta_{N}(t-y) d y \quad s>t-\tau .
\end{array}\right.
$$

Our goal is to build a compact uniformly attracting set for the process. In the sequel, let $\rho>0$ be fixed such that $B_{\mathcal{H}}(0, \rho)$ is a uniformly absorbing set in $\mathcal{H}$ for $U_{h}(t, \tau)$, as $h \in F$ (whose existence is assured by Theorem 3.2). Moreover, with reference to Definition 3.1, let $t_{\rho}=t^{*}\left(B_{\mathcal{H}}(0, \rho)\right)$.

Lemma 4.5. Assume (h1)-(h3), and let (g1)-(g3) with $\beta \leq \frac{5}{3}$ hold. Let $F$ and $\Phi$ be as in Theorem 3.3. Then there exists a positive constant $\Gamma$, depending on $\Phi$, such that

$$
\left\|z_{N}(t)\right\|_{\mathcal{V}}^{2} \leq \Gamma\left(1+\left\|z\left(t_{0}\right)\right\|_{\mathcal{H}}\right)^{4} \quad \forall t \geq t_{0},
$$


for any $t_{0} \geq \tau, \tau \in \mathbb{R}$. Moreover, such a $\Gamma$ does not depend on the particular initial time $\tau$ chosen.

Proof. We parallel the proof of Theorem 3.3 (with $z_{N}$ in place of $z$ ), the only difference being the evaluation of the term $2\left\langle g(\theta), \Delta \theta_{N}\right\rangle$. Indeed in this case, using Young's inequality, (g1), and Lemma 1.3, we obtain

$$
\begin{aligned}
2\left\langle g(\theta), \Delta \theta_{N}\right\rangle & \leq \frac{k_{0}}{2}\left\|\Delta \theta_{N}\right\|^{2}+\frac{2 c_{1}^{2}}{k_{0}}\left\|1+|\theta|^{\beta}\right\|^{2} \\
& \leq \frac{k_{0}}{2}\left\|\Delta \theta_{N}\right\|^{2}+c_{7}+c_{8}\|\theta\|_{2 \gamma+2}^{2 \gamma+2} \\
& \leq \frac{k_{0}}{2}\left\|\Delta \theta_{N}\right\|^{2}+c_{7}+c c_{8}\|\nabla \theta\|^{3 \gamma}\|\theta\|^{2-\gamma} \\
& \leq \frac{k_{0}}{2}\left\|\Delta \theta_{N}\right\|^{2}+c_{7}+c c_{8}\left(1+\|\nabla \theta\|^{2}\right)\left(1+\|\theta\|^{2}\right)
\end{aligned}
$$

since $\beta \leq \frac{5}{3}$. Thus, denoting

$$
\varphi=\frac{2}{k_{0}}\|h\|^{2}+c_{7}+c c_{8}\left(1+\|\nabla \theta\|^{2}\right)\left(1+\|\theta\|^{2}\right)
$$

we conclude that

$$
\frac{d}{d t}\left\|z_{N}\right\|_{\mathcal{V}}^{2}+\varepsilon_{1}\left\|z_{N}\right\|_{\mathcal{V}}^{2} \leq \varphi
$$

From (3.3) and (3.5) there exist two positive constants $K_{3}$ and $K_{4}$ (depending on $\Phi$ ) such that

$$
\int_{t}^{t+1} \varphi(y) d y \leq K_{3}\left\|z\left(t_{0}\right)\right\|_{\mathcal{H}}^{4}+K_{4} \quad \forall t \geq t_{0},
$$

for any given $t_{0} \geq \tau$. Hence Lemma 1.4 and Lemma 1.5 lead to

$$
\left\|z_{N}(t)\right\|_{\mathcal{V}}^{2} \leq C\left(\varepsilon_{1}\right)\left(K_{3}\left\|z\left(t_{0}\right)\right\|_{\mathcal{H}}^{4}+K_{4}\right)
$$

as claimed.

Lemma 4.6. Assume (h1)-(h3), and (g1)-(g3) with $\beta \leq \frac{5}{3}$, and let $F$ and $\Phi$ be as in Theorem 3.3. Denote

$$
\mathcal{M}=\bigcup_{h \in F} \bigcup_{z_{0} \in B_{\mathcal{H}}(0, \rho)} \bigcup_{\tau \in \mathbb{R}} \bigcup_{t \geq \tau+t_{\rho}} \eta_{N}^{t}\left(\cdot ; \tau, z_{0}, h\right) .
$$

Then $\mathcal{M}$ is relatively compact in $L_{\mu}^{2}\left(\mathbb{R}^{+}, H_{0}^{1}\right)$.

Proof. It is clear from Lemma 4.5 that $\mathcal{M}$ is bounded in $L_{\tau}^{2}\left(\mathbb{R}^{+}, H^{2} \cap H_{0}^{1}\right)$. Let then $\eta_{N}^{t} \in \mathcal{M}$. The derivative of (4.8) yields

$$
\frac{\partial}{\partial s} \eta_{N}^{t}(s)= \begin{cases}\theta_{N}(t-s) & 0 \leq s \leq t-\tau \\ 0 & s>t-\tau\end{cases}
$$

Thus (4.7) and (4.10) entail

$$
\int_{0}^{\infty} \mu(s)\left\|\frac{\partial}{\partial s} \eta_{N}^{t}(s)\right\|^{2} d s=\int_{0}^{t-\tau} \mu(s)\left\|\theta_{N}(t-s)\right\|^{2} d s \leq\left(8 \rho^{2}+2 \Psi\right)\|\mu\|_{L^{1}\left(\mathbb{R}^{+}\right)}
$$


So we conclude that $\mathcal{M}$ is bounded in $L_{\mu}^{2}\left(\mathbb{R}^{+}, H^{2} \cap H_{0}^{1}\right) \cap H_{\mu}^{1}\left(\mathbb{R}^{+}, L^{2}\right)$. Moreover, from (4.8) and (4.9), with $t_{0}=\tau$, it is easy to check that for every $\eta \in \mathcal{M}$,

$$
\|\nabla \eta(s)\|^{2} \leq s^{2} \Gamma(1+\rho)^{4} \in L_{\mu}^{1}\left(\mathbb{R}^{+}\right)
$$

by force of the exponential decay of $\mu$. The proof is completed applying the following result from $[21]$ :

Let $\mu \in C\left(\mathbb{R}^{+}\right) \cap L^{1}\left(\mathbb{R}^{+}\right)$be a nonnegative function, such that if $\mu\left(s_{0}\right)=0$ for some $s_{0} \in \mathbb{R}^{+}$, then $\mu(s)=0$ for every $s>s_{0}$. Let $B_{0}, B, B_{1}$ be three Banach spaces, with $B_{0}$ and $B_{1}$ reflexive, such that

$$
B_{0} \hookrightarrow B \hookrightarrow B_{1},
$$

the first injection being compact. Let $\mathcal{M} \subset L_{\mu}^{2}\left(\mathbb{R}^{+}, B\right)$ satisfy the following hypotheses:

(i) $\mathcal{M}$ is bounded in $L_{\mu}^{2}\left(\mathbb{R}^{+}, B_{0}\right) \cap H_{\mu}^{1}\left(\mathbb{R}^{+}, B_{1}\right)$,

(ii) $\sup _{\eta \in \mathcal{M}}\|\eta(s)\|_{B}^{2} \leq h(s) \quad \forall s \in \mathbb{R}^{+}$for some $h \in L_{\mu}^{1}\left(\mathbb{R}^{+}\right)$.

Then $\mathcal{M}$ is relatively compact in $L_{\mu}^{2}\left(\mathbb{R}^{+}, B\right)$.

Proposition 4.7. Assume (h1)-(h3), and let (g1)-(g3) with $\beta \leq \frac{5}{3}$ hold. Let $F$ and $\Phi$ be as in Theorem 3.3. Then there exists a compact, uniformly attracting set for the family $\left\{U_{h}(t, \tau), h \in F\right\}$.

Proof. Denote by $\overline{\mathcal{M}}$ the closure of $\mathcal{M}$ in $L_{\mu}^{2}\left(\mathbb{R}^{+}, H_{0}^{1}\right)$, and introduce the set

$$
\mathcal{K}=B_{H^{2} \cap H_{0}^{1}}\left(0, \Gamma(1+\rho)^{4}\right) \times \overline{\mathcal{M}} \subset \mathcal{H} .
$$

From the compact embedding $H^{2} \cap H_{0}^{1} \hookrightarrow H_{0}^{1}$ and Lemma $4.6, \mathcal{K}$ is compact in $\mathcal{H}$. We need to show the uniform attraction property. Let $\mathcal{B} \subset \mathcal{H}$ be a bounded set, with $R=\sup _{z \in \mathcal{B}}\|z\|_{\mathcal{H}}$, and let $t^{*}=t^{*}(\mathcal{B})$ be such that, for every $h \in F$,

$$
U_{h}(t, \tau) \mathcal{B} \subset B_{\mathcal{H}}(0, \rho) \quad \forall t \geq \tau+t^{*} .
$$

Let then $t>t_{\rho}+t^{*}+\tau$, and set $\hat{t}=t-t_{\rho}-t^{*}-\tau>0$. Using the process properties we get that

$$
\begin{aligned}
U_{h}\left(\hat{t}+t_{\rho}+t^{*}+\tau, \tau\right) \mathcal{B} & =U_{h}\left(\hat{t}+t_{\rho}+t^{*}+\tau, t^{*}+\tau\right) U_{h}\left(t^{*}+\tau, \tau\right) \mathcal{B} \\
& \subset U_{h}\left(\hat{t}+t_{\rho}+t^{*}+\tau, t^{*}+\tau\right) B_{\mathcal{H}}(0, \rho) .
\end{aligned}
$$

Pick any $z(t) \in U_{h}(t, \tau) \mathcal{B}$, for $t>t_{\rho}+t^{*}+\tau$. Applying (4.9) with $t_{0}=\tau+t^{*}$, we get

$$
\left\|\Delta \theta_{N}(t)\right\|^{2} \leq\left\|z_{N}(t)\right\|_{\mathcal{V}}^{2} \leq \Gamma\left(1+\left\|z\left(\tau+t^{*}\right)\right\|_{\mathcal{H}}\right)^{4} \leq \Gamma(1+\rho)^{4} .
$$

It is then apparent that $z_{N}(t) \in \mathcal{K}$. Therefore, from (4.6),

$$
\inf _{m \in \mathcal{K}}\|z(t)-m\|_{\mathcal{H}} \leq\left\|z_{L}(t)\right\|_{\mathcal{H}} \leq \sqrt{2} R e^{-\frac{\varepsilon_{0}}{2}(t-\tau)} \quad \forall t>t_{\rho}+t^{*}+\tau
$$

The above inequality being independent of $h \in F$, we conclude that

$$
\sup _{h \in F^{*}} \operatorname{dist}\left(U_{h}(t, \tau) \mathcal{B}, \mathcal{K}\right) \leq \sqrt{2} R e^{-\frac{\varepsilon_{0}}{2}(t-\tau)} \quad \forall t>t_{\rho}+t^{*}+\tau .
$$

Hence (4.1) holds, and the result is proved.

We are now ready to state the main result of the section. 
Theorem 4.8. Assume (h1)-(h3), and let (g1)-(g3) with $\beta \leq \frac{5}{3}$ hold. Furthermore, let

$$
f \in \mathcal{T}_{c}^{2}\left(\mathbb{R}, L^{2}\right)
$$

Then there exists a compact uniform attractor $\mathcal{A}$ for the family $\left\{U_{h}(t, \tau), h \in H(f)\right\}$ given by

$$
\mathcal{A}=\left\{\begin{array}{l}
z(0) \text { such that } z(t) \text { is any bounded complete } \\
\text { trajectory of } U_{h}(t, \tau) \text { for some } h \in H(f)
\end{array}\right\} .
$$

Proof. We apply Theorem 4.3 with $F=H(f)$ and

$$
T(t) h(s)=h(s+t), \quad h \in H(f)
$$

(i.e., $T(t)$ is the translation semigroup acting on $H(f))$. It is then immediate to verify the translation equality (4.2). From Proposition 4.7, with $\Phi=\|f\|_{\mathcal{T}_{b}^{2}\left(\mathbb{R} . L^{2}\right)}$, we get that the family $\left\{U_{h}(t, \tau), h \in H(f)\right\}$ is uniformly asymptotically compact. The proof of the $(\mathcal{H} \times H(f), \mathcal{H})$-continuity is practically identical to the proof of Theorem 2.2, and is therefore omitted.

Notice that the attractor of the semigroup $T(t)$ on $H(f)$ coincides with the entire space $H(f)$.

In the course of the investigation, we showed that $B_{\mathcal{H}}(0, \rho) \times H(f)$ is a bounded absorbing set for the semigroup $S(t)$ defined in (4.3). In particular, $B_{\mathcal{H}}(0, \rho) \times H(f)$ is connected in $\mathcal{H} \times H(f)$. Indeed, it is immediate to see that $\{f(\cdot+r)\}_{r \in \mathbb{R}}$ is path connected, and therefore its closure, i.e., $H(f)$ is connected. Then by [17], Proposition 5.2.7, the attractor $\widetilde{\mathcal{A}}$ of $S(t)$ is connected, and so is its projection on $\mathcal{H}$. We summarize this discussion in the next corollary.

Corollary 4.9. The uniform attractor $\mathcal{A}$ for the family $\left\{U_{h}(t, \tau), h \in H(f)\right\}$ given by Theorem 4.8 is connected.

REMARK 4.10. The restriction $\beta \leq \frac{5}{3}$ is due to the presence of the memory. In fact, in the particular case when the memory kernel vanishes, we reduce to the semilinear equation

$$
\theta_{t}-k_{0} \Delta \theta+g(\theta)=h
$$

and, using the uniform Gronwall lemma (see, e.g., [22]), it is easy to show that the above results hold for $\beta<\frac{7}{3}$.

5. Hausdorff Dimension of the Uniform Attractor. In the previous Theorem 4.3 and Theorem 4.4 it is shown that the uniform attractor $\mathcal{A}$ of the family $\left\{U_{h}(t, \tau), h \in\right.$ $H(f)\}$ is the projection on $\mathcal{H}$ of the attractor $\widetilde{\mathcal{A}}$ of the corresponding semigroup $S(t)$, acting on $\mathcal{H} \times H(f)$. Therefore, the Hausdorff dimensions of these sets satisfy the inequality $\operatorname{dim}_{\mathrm{H}} \mathcal{A} \leq \operatorname{dim}_{\mathrm{H}} \tilde{\mathcal{A}}$. In this section, along the line of [5], we show that $\operatorname{dim}_{\mathrm{H}} \widetilde{\mathcal{A}}<\infty$ (and thus $\left.\operatorname{dim}_{\mathrm{H}} \mathcal{A}<\infty\right)$ when the external heat source has a quasiperiodic dependence on time. 
We recall that the Hausdorff dimension of a subset $\mathcal{X}$ of a metric space $\mathcal{E}$ is defined by

$$
\operatorname{dim}_{\mathrm{H}} \mathcal{X}=\sup \left\{d>0: \sup _{\varepsilon>0} \inf _{C_{\varepsilon}} \sum_{i \in I} r_{i}^{d}<+\infty\right\}
$$

where $C_{\varepsilon}=\left\{B_{i}\left(r_{i}\right)\right\}_{i \in I}$ is a covering of $\mathcal{X}$ by balls of radii $r_{i} \leq \varepsilon$.

We now state the fundamental result from [10] concerning the Hausdorff dimensions of fully invariant sets. We first need two definitions.

Definition 5.1. Let $\mathcal{E}$ be a Hilbert space, $\mathcal{L}(\mathcal{E})$ the space of continuous linear operators from $\mathcal{E}$ to $\mathcal{E}, \mathcal{X} \subset \mathcal{E}$, and $S$ a (nonlinear) continuous map from $\mathcal{X}$ into $\mathcal{E}$. Then $S$ is said to be uniformly quasidifferentiable on $\mathcal{X}$ if for any $u \in \mathcal{X}$ there exists $S^{\prime}(u) \in \mathcal{L}(\mathcal{E})$ (the quasidifferential of $S$ at $u$ with respect to $\mathcal{X}$ ) such that

$$
\left\|S u-S v-S^{\prime}(u)(u-v)\right\|_{\mathcal{E}} \leq \sigma\left(\|u-v\|_{\mathcal{E}}\right)\|u-v\|_{\mathcal{E}} \quad \forall v \in \mathcal{X},
$$

where $\sigma: \mathbb{R} \rightarrow \mathbb{R}^{+}$is independent on $u$, and $\sigma(y) \rightarrow 0$ as $y \rightarrow 0^{+}$. The operator $S^{\prime}(u)$ might not be unique.

Definition 5.2. Let $M$ be a linear operator on a Hilbert space $\mathcal{E}$. For any $m \in \mathbb{N}$ the $m$-dimensional trace of $M$ is defined as

$$
\operatorname{Tr}_{m} M=\sup _{Q} \sum_{j=1}^{m}\left\langle M u_{j}, u_{j}\right\rangle_{\mathcal{E}}
$$

where the supremum ranges over all possible orthogonal projections $Q$ in $\mathcal{E}$ on the $m$ dimensional space $Q \mathcal{E}$ belonging to the domain of $M$, and $\left\{u_{1}, \ldots, u_{m}\right\}$ is an orthonormal basis of $Q \mathcal{E}$.

The following result holds.

TheOREM 5.3. Let there be given a Hilbert space $\mathcal{E}$, and let $\mathcal{X} \subset \mathcal{E}$ be a compact fully invariant set for $S(t)$, i.e., $S(t) \mathcal{X}=\mathcal{X}$ for all $t \geq 0$. Assume also that $S(t)$ is uniformly quasidifferentiable on $\mathcal{X}$ for all $t \geq 0$, and

$$
\sup _{u_{0} \in \mathcal{X}}\left\|S^{\prime}\left(t, u_{0}\right)\right\|_{L(\mathcal{E})} \leq C(t)<\infty \quad \forall t \geq 0
$$

where $S^{\prime}\left(t, u_{0}\right)$ is the quasidifferential of $S(t)$ at $u_{0}$. It is also assumed that $S^{\prime}\left(t, u_{0}\right)$ is generated by the equation in variation

$$
\begin{aligned}
U_{t} & =M(u) U, \\
U(0) & =U_{0},
\end{aligned}
$$

that is, $S^{\prime}(t, u) U_{0}=U(t)$ with $u(t)=S(t) u_{0}$. Introducing the number $q_{m}$ by the formula

$$
q_{m}=\liminf _{T \rightarrow \infty} \sup _{u \in \mathcal{X}}\left\{\frac{1}{T} \int_{0}^{T} \operatorname{Tr}_{m} M(S(t) u) d t\right\},
$$

if there exists $m$ such that $q_{m}<0$, then $\operatorname{dim}_{\mathrm{H}} \mathcal{X} \leq m$.

As anticipated at the beginning of the section, we shall consider a quasiperiodic external heat supply, i.e., a function $f: \Omega \times \mathbb{R} \rightarrow \mathbb{R}$ of the form

$$
f(x, t)=\Phi(x, \Lambda t)=\Phi\left(x, \lambda_{1} t, \ldots, \lambda_{K} t\right)
$$


where $\Phi(x, \omega) \in C^{1}\left(\mathbf{T}^{K}, L^{2}\right)$ is a $2 \pi$-periodic function of $\omega$ on the $K$-dimensional torus $\mathbf{T}^{K}$, and $\Lambda=\left(\lambda_{1}, \ldots, \lambda_{K}\right)$ are rationally independent numbers. For further reference we agree to denote

$$
\Phi^{\prime}(x, \omega)=\left(\frac{\partial}{\partial \omega_{1}} \Phi(x, \omega), \ldots, \frac{\partial}{\partial \omega_{K}} \Phi(x, \omega)\right), \quad \omega=\left(\omega_{1}, \ldots, \omega_{K}\right) .
$$

It is immediate to check that $f \in \mathcal{T}_{c}^{2}(\mathbb{R}, L)$, and $h \in H(f)$ if and only if

$$
h(x, t)=\Phi\left(x, \Lambda t+\omega_{0}\right), \quad \omega_{0} \in \mathbf{T}^{K} .
$$

Therefore, $H(f)$ might be identified with $\mathbf{T}^{K}$, and the translation semigroup acting on $H(f)$ is equivalent to the translation semigroup $T(t)$ on $\mathbf{T}^{K}$, defined by

$$
T(t) \omega_{0}=\left[\Lambda t+\omega_{0}\right]=\left(\Lambda t+\omega_{0}\right)(\bmod 2 \pi)^{K} .
$$

In the remainder of the paper we shall denote $\mathcal{E}=\mathcal{H} \times \mathbf{T}^{K}$, and we consider the semigroup $S(t)$ acting on $\mathcal{E}$ given by (4.3), with $T(t)$ as above. Clearly, with reference to the previous section, the set $\widetilde{\mathcal{A}}=\mathcal{A} \times \mathbf{T}^{K}(\mathcal{A}$ being the uniform attractor of the family $\left.\left\{U_{\omega}(t, \tau), \omega \in \mathbf{T}^{K}\right\}\right)$ is the attractor of $S(t)$. For every $w_{0}=\left(z_{0}, \omega_{0}\right) \in \mathcal{E}$, the vector $w(t)=S(t) w_{0}=(z(t), \omega(t))$ is the solution of the differential equation

$$
\begin{aligned}
z_{t} & =L z+G(z, \omega), \\
\omega_{t} & =\Lambda, \\
z(0) & =z_{0}, \\
\omega(0) & =\omega_{0},
\end{aligned}
$$

where

$$
G(z, \omega)=(\Phi(\omega)-g(\theta), 0)
$$

In the above formula and in the sequel, without further warning, we use the complete decomposition of $w$, i.e., $w=(\theta, \eta, \omega)=\left(\Pi_{1} w, \Pi_{2} w, \Pi_{3} w\right)$, where $\Pi_{j}, j=1,2,3$, denote the projections on $L^{2}, L_{\mu}^{2}\left(\mathbb{R}^{+}, H_{0}^{1}\right)$, and $\mathbf{T}^{K}$, respectively. We also define the operators $A$ (linear) and $F$ on $\mathcal{E}$ by $A w=(L z, 0)$ and $F(w)=(G(z, \omega), 0)$.

Theorem 5.4. Assume (h1)-(h2), and let (g1)-(g3) with $\beta \leq \frac{7}{3}$ and $g \in C^{1}(\mathbb{R})$ hold. Then the semigroup $S(t)$ acting on $\mathcal{E}$ is uniformly quasidifferentiable on any bounded set $\mathcal{X}$ that is invariant for $S(t)$, i.e., $S(t) \mathcal{X} \subset \mathcal{X}$ for every $t \geq 0$, and the quasidifferential $S^{\prime}\left(t, w_{0}\right)$ at the point $w_{0}=\left(z_{0}, \omega_{0}\right)=\left(\theta_{0}, \eta_{0}, \omega_{0}\right)$ satisfies the variation equation

$$
\begin{aligned}
& W_{t}=A W+F^{\prime}(w) W, \\
& W(0)=W_{0}=\left(Z_{0}, \Sigma_{0}\right)=\left(\Theta_{0}, H_{0}, \Sigma_{0}\right),
\end{aligned}
$$

where

$$
\begin{gathered}
S^{\prime}\left(t, w_{0}\right) W_{0}=W(t)=(Z(t), \Sigma(t))=\left(\Theta(t), H^{t}, \Sigma(t)\right), \\
w(t)=(z(t), \omega(t))=\left(\theta(t), \eta^{t}, \omega(t)\right)=S(t) w_{0},
\end{gathered}
$$

and

$$
F^{\prime}(w) W=\left(-g^{\prime}(\theta) \Theta+\Phi^{\prime}(\omega) \Sigma, 0,0\right)
$$


$F^{\prime}$ being the Fréchet differential of $F$. Furthermore,

$$
\sup _{w_{0} \in \mathcal{X}}\left\|S^{\prime}\left(t, w_{0}\right)\right\|_{L(\mathcal{E})} \leq C(t)<\infty \quad \forall t \geq 0 .
$$

Proof. Let $w=(z, \omega)$ and $w^{*}=\left(z^{*}, \omega^{*}\right)$ be solutions to system (5.1) with initial data $w_{0}$ and $w_{0}^{*}$, respectively, with $w_{0}, w_{0}^{*} \in \mathcal{X}$. The difference $\widetilde{w}=w^{*}-w$ satisfies the problem

$$
\begin{aligned}
\tilde{w}_{t} & =A \tilde{w}+F\left(w^{*}\right)-F(w), \\
\tilde{w}(0) & =\tilde{w}_{0}=w_{0}^{*}-w_{0} .
\end{aligned}
$$

Arguing as in the proof of Theorem 2.2, taking the inner product in $\mathcal{E}$ of $(5.3)$ and $\tilde{w}$, we get

$$
\frac{d}{d t}\|\tilde{w}\|_{\mathcal{E}}^{2} \leq m_{\gamma}\|\tilde{w}\|_{\mathcal{E}}^{2}+2\left|\left\langle\Phi\left(\omega^{*}\right)-\Phi(\omega), \tilde{\theta}\right\rangle\right|
$$

with $m_{\gamma}$ given by (2.5) if $\gamma \leq 1$, and by (2.8) if $1<\gamma \leq \frac{4}{3}$. Due to the fact that $\mathcal{X}$ is invariant for $S(t)$ and is bounded in $\mathcal{E}$, from (2.2) it is clear that

$$
\sup _{\theta^{*} . \theta \in \Pi_{1} \mathcal{X}} m_{\gamma} \in L^{1}([0, T]) \quad \forall T>0
$$

Since

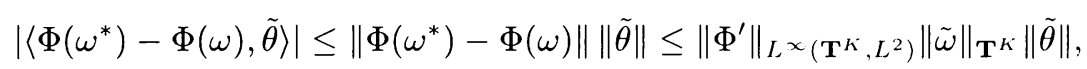

using Young's inequality and defining $r_{\gamma}=m_{\gamma}+\left\|\Phi^{\prime}\right\|_{L^{\infty}\left(\mathbf{T}^{K}, L^{2}\right)}$, by virtue of Gronwall's lemma we conclude that

$$
\|\tilde{w}(t)\|_{\mathcal{E}}^{2} \leq\left\|\tilde{w}_{0}\right\|_{\mathcal{E}}^{2} \exp \left[\int_{0}^{t} r_{\gamma}(y) d y\right] \leq\left\|\tilde{w}_{0}\right\|_{\mathcal{E}}^{2} C(T) \quad \forall t \leq T,
$$

where $C(T)<\infty$ for all $T>0$. Let now $W$ be the solution to (5.2) with initial data $W(0)=\tilde{w}_{0}$. Indeed, it is easy to see that the linear (non-autonomous) problem (5.2) with initial data $W(0)=\tilde{w}_{0}$ possesses a unique solution $W \in C([0, T], \mathcal{E})$ for all $T>0$. Our goal is to show that $W(t)=S^{\prime}\left(t, w_{0}\right) \tilde{w}_{0}$. Denote

$$
\varphi=w^{*}-w-W=\tilde{w}-W .
$$

Clearly $\varphi$ satisfies

$$
\begin{aligned}
& \varphi_{t}=A \varphi+F^{\prime}(w) \varphi+h\left(w^{*}, w\right) \\
& \varphi(0)=0
\end{aligned}
$$

where

$$
h\left(w^{*}, w\right)=F\left(w^{*}\right)-F(w)-F^{\prime}(w) \tilde{w} .
$$

Due to the differentiability assumptions on $g$ and $\Phi$, there exists $\sigma: \mathbb{R} \rightarrow \mathbb{R}^{+}, \sigma(y) \rightarrow 0$ as $y \rightarrow 0^{+}$, such that

$$
\frac{\left\|h\left(w^{*}, w\right)\right\|_{\mathcal{E}}}{\|\tilde{w}\|_{\mathcal{E}}}=\sigma\left(\|\tilde{w}\|_{\mathcal{E}}\right)
$$

We take the inner product in $\mathcal{E}$ of (5.5) and $\varphi$. It is easily seen that the estimate for the term $\left\langle F^{\prime}(w) \varphi, \varphi\right\rangle_{\mathcal{E}}$ can be carried out exactly as above, whereas, by Young's inequality,

$$
2\left\langle h\left(w^{*}, w\right), \varphi\right\rangle_{\mathcal{E}} \leq\left\|h\left(w^{*}, w\right)\right\|_{\mathcal{E}}^{2}+\|\varphi\|_{\mathcal{E}}^{2} .
$$


Thus we get

$$
\frac{d}{d t}\|\varphi\|_{\mathcal{E}}^{2} \leq\left(r_{\gamma}+1\right)\|\varphi\|_{\mathcal{E}}^{2}+\left\|h\left(w^{*}, w\right)\right\|_{\mathcal{E}}^{2}
$$

Gronwall's lemma and (5.4) entail

$$
\begin{aligned}
\|\varphi(t)\|_{\mathcal{E}}^{2} & \leq\left[\int_{0}^{t}\left\|h\left(w^{*}(y), w(y)\right)\right\|_{\mathcal{E}}^{2} d y\right] \exp \left[\int_{0}^{t}\left(r_{\gamma}(y)+1\right) d y\right] \\
& \leq \sup _{s \in\left[0, T^{\prime}\right]} \sigma^{2}\left(\|\widetilde{w}(s)\|_{\mathcal{E}}\right)\left[\int_{0}^{t}\|\tilde{w}(y)\|_{\mathcal{E}}^{2} d y\right] e^{T} C(T) \\
& \leq \sup _{s \in[0, T]} \sigma^{2}\left(\|\tilde{w}(s)\|_{\mathcal{E}}\right)\left\|\tilde{w}_{0}\right\|_{\mathcal{E}}^{2} \widetilde{C}(T) \quad \forall t \leq T
\end{aligned}
$$

with $\widetilde{C}(T)=T e^{T}(C(T))^{2}$. From (5.4) we also get that

$$
\lim _{\tilde{w}_{0} \rightarrow 0} \sup _{s \in\left[0, T^{\prime}\right]} \sigma^{2}\left(\|\tilde{w}(s)\|_{\mathcal{E}}\right)=0 .
$$

Then we conclude that, for every $T>0$,

$$
\lim _{\tilde{w}_{0} \rightarrow 0} \frac{\|\varphi(T)\|_{\mathcal{E}}^{2}}{\left\|\tilde{w}_{0}\right\|_{\mathcal{E}}^{2}}=0,
$$

which gives the required uniform quasidifferentiability. Finally, taking the inner product of (5.2) and $W$, and performing calculations analogous to those leading to (5.6), we obtain the estimate

$$
\|W(T)\|_{\mathcal{E}}^{2} \leq\left\|W_{0}\right\|_{\mathcal{E}}^{2} C(T) \quad \forall T \geq 0
$$

which yields the last assertion of the theorem.

It is immediate to verify that Theorem 5.4 still holds if we replace condition (g3) with $\beta \leq \frac{7}{3}$ with condition ( $\mathrm{g} 4$ ).

We now state our result about the dimension of the attractor.

Theorem 5.5. Assume (h1)-(h2), and let (g1)-(g3) with $\beta \leq \frac{5}{3}$ and $g \in C^{1}(\mathbb{R})$ hold. Then the attractor $\widetilde{\mathcal{A}}$ of the semigroup $S(t)$ acting on $\mathcal{E}$ has finite Hausdorff dimension.

Proof. Let $w_{0} \in \widetilde{\mathcal{A}}$ (so that $w \in \widetilde{\mathcal{A}}$ ). Let $W=(\Theta, H, \Sigma)$ be a unitary vector belonging to the domain of $A+F^{\prime}(w)$. Then

$$
\left\langle\left(A+F^{\prime}(w)\right) W, W\right\rangle_{\mathcal{E}} \leq\langle A W, W\rangle_{\mathcal{E}}-\left\langle g^{\prime}(\theta) \Theta, \Theta\right\rangle+\left\langle\Phi^{\prime}(\omega) \Sigma, \Theta\right\rangle .
$$

From a direct calculation (see also [14]),

$$
\langle A W, W\rangle_{\mathcal{E}} \leq-k_{0}\|\nabla \Theta\|^{2}-\frac{\delta}{2}\|\nabla H\|_{\mu}^{2}
$$

An application of the generalized Hölder inequality entails

$$
-\left\langle g^{\prime}(\theta) \Theta, \Theta\right\rangle \leq\|\Theta\|_{6}\|\Theta\|\left\|g^{\prime}(\theta)\right\|_{3} .
$$

Since $\beta \leq 5 / 3$, the term $\left\|g^{\prime}(\theta)\right\|_{3}^{2}$ is uniformly bounded as $w_{0} \in \widetilde{\mathcal{A}}$. Thus, exploiting the embedding $H_{0}^{1} \hookrightarrow L^{6}$, and using Young's inequality, we get

$$
-\left\langle g^{\prime}(\theta) \Theta, \Theta\right\rangle \leq \frac{k_{0}}{2}\|\nabla \Theta\|^{2}+c_{9}\|\Theta\|^{2}
$$


for some $c_{9}>0$. Finally, since $\left\|\Phi^{\prime}\right\|_{L^{x}\left(\mathbf{T}^{K}, L^{2}\right)}<\infty$,

$$
\left\langle\Phi^{\prime}(\omega) \Sigma, \Theta\right\rangle \leq c_{10}\|\Theta\|^{2}+c_{10}\|\Sigma\|_{\mathbf{T}^{K}}^{2}
$$

for some $c_{10}>0$. Adding the pieces together, setting $c_{11}=c_{9}+c_{10}$, we have

$$
\left\langle\left(A+F^{\prime}(w)\right) W, W\right\rangle_{\mathcal{E}} \leq-\frac{k_{0}}{2}\|\nabla \Theta\|^{2}-\frac{\delta}{2}\|\nabla H\|_{\mu}^{2}+c_{11}\|\Theta\|^{2}+c_{10}\|\Sigma\|_{\mathbf{T}^{K}}^{2} .
$$

Therefore, we conclude that $A+F^{\prime}(w) \leq M$, where $M$ is the diagonal operator acting on $L^{2} \oplus L_{\mu}^{2}\left(\mathbb{R}^{+}, H_{0}^{1}\right) \oplus \mathbf{T}^{K}$ defined by

$$
M=\left(\begin{array}{ccc}
\frac{k_{0}}{2} \Delta+c_{11} I & 0 & 0 \\
0 & -\frac{\delta}{2} I & 0 \\
0 & 0 & c_{10} I
\end{array}\right) .
$$

From the definition of $\operatorname{Tr}_{m}$, it is apparent that $\operatorname{Tr}_{m}\left(A+F^{\prime}(w)\right) \leq \operatorname{Tr}_{m}(M)$. Since $M$ is diagonal, it is easy to see that

$$
\operatorname{Tr}_{m}(M)=\sup _{Q} \sum_{j=1}^{m}\left\langle M W_{j}, W_{j}\right\rangle_{\mathcal{E}}
$$

where the supremum is taken over the projections $Q$ of the form $Q_{1} \oplus Q_{2} \oplus Q_{3}$. This amounts to considering vectors $W_{j}$ where only one of the three components is nonzero (and in fact of norm one in its space). Choose then $m>K$, and let $n_{1}, n_{2}, n_{3}$ be the numbers of vectors $W_{j}$ of the form $(\Theta, 0,0),(0, H, 0)$, and $(0,0, \Sigma)$, respectively. Notice that, since $\mathbf{T}^{K}$ is $K$-dimensional, $n_{3} \leq K$. Thus, applying Lemma 1.6, we get

$$
\operatorname{Tr}_{m}(M) \leq-\frac{k_{0}}{2} \kappa n_{1}^{\frac{5}{3}}+c_{11} n_{1}-\frac{\delta}{2} n_{2}+c_{10} n_{3}
$$

which gives at once

$$
q_{m} \leq-\frac{k_{0}}{2} \kappa n_{1}^{\frac{5}{3}}+c_{11} n_{1}-\frac{\delta}{2} n_{2}+c_{10} K
$$

Since as $m$ goes to infinity, either $n_{1}$ or $n_{2}$ (or both) go to infinity, it is clear that there exists $m_{0}$ such that $q_{m_{0}}<0$. Thus, the desired conclusion follows from Theorem 5.3 and Theorem 5.4.

Corollary 5.6. Assume (h1)-(h2), and let (g1)-(g3) with $\beta \leq \frac{5}{3}$ and $g \in C^{1}(\mathbb{R})$ hold. Let $f \in C^{1}\left(\mathbb{R}, L^{2}\right)$ be quasiperiodic in time. Then the uniform attractor $\mathcal{A}$ of the family $\left\{U_{h}(t, \tau), h \in H(f)\right\}$ given by Theorem 4.7 has finite Hausdorff dimension.

\section{REFERENCES}

[1] S. Aizicovici and V. Barbu, Existence and asymptotic results for a system of integro-partial differential equations, NoDEA Nonlinear Differential Equations Appl. 3, 1-18 (1996)

[2] L. Amerio and G. Prouse, Abstract almost periodic functions and functional equations, Van Nostrand, New York, 1971

[3] G. Bonfanti, P. Colli, M. Grasselli, and F. Luterotti, Nonsmooth kernels in a phase relaxation problem with memory, Nonlinear Anal. 32, 455-465 (1998)

[4] H. Brézis, Operateurs maximaux monotones, North-Holland, Amsterdam, 1973

[5] V. V. Chepyzhov and M. I. Vishik, Nonautonomous evolution equations and their attractors, Russian J. Math. Phys. 1, 165-190 (1993)

[6] V. V. Chepyzhov and M. I. Vishik, Attractors of non-autonomous dynamical systems and their dimension, J. Math. Pures Appl. 73, 279-333 (1994) 
[7] V. V. Chepyzhov and M. I. Vishik, Non-autonomous evolutionary equations with translation compact symbols and their attractor, C. R. Acad. Sci. Paris Sér. I Math. 321, 153-158 (1995)

[8] B. D. Coleman and M. E. Gurtin, Equipresence and constitutive equation for rigid heat conductors, Z. Angew. Math. Phys. 18, 199-208 (1967)

[9] P. Colli and M. Grasselli, Phase transition problems in materials with memory, J. Integral Equations Appl. 5, 1-22 (1993)

[10] P. Constantin, C. Foias, and R. Temam, Attractors representing turbulent flows, Mem. Amer. Math. Soc. 53, no. 314 (1993)

[11] C. M. Dafermos, Asymptotic stability in viscoelasticity, Arch. Rational Mech. Anal. 37, 297-308 (1970)

[12] W. Desch and R. K. Miller, Exponential stabilization of Volterra integrodifferential equations in Hilbert spaces, J. Differential Equations 70, 366-389 (1987)

[13] G. Gentili and C. Giorgi, Thermodynamic properties and stability for the heat flux equation with linear memory, Quart. Appl. Math. 51, 343-362 (1993)

[14] C. Giorgi, A. Marzocchi, and V. Pata, Asymptotic behavior of a semilinear problem in heat conduction with memory, NoDEA Nonlinear Differential Equations Appl. 5, 333-354 (1998)

[15] H. Grabmüller, On linear theory of heat conduction in materials with memory. Existence and uniqueness theorems for the final value problem, Proc. Roy. Soc. Edinburgh A-76, 119-137 (197677)

[16] M. Grasselli and V. Pata, Longtime behavior of a homogenized model in viscoelastodynamics, Discrete Contin. Dynam. Systems 4, 339-358 (1998)

[17] A. Haraux, Systèmes dynamiques dissipatifs et applications, Masson, Paris, 1990

[18] R. K. Miller, An integrodifferential equation for rigid heat conductors with memory, J. Math. Anal. Appl. 66, 331-332 (1978)

[19] J. W. Nunziato, On heat conduction in materials with memory, Quart. Appl. Math. 29, 187-204 (1971)

[20] V. Pata, G. Prouse, and M. I. Vishik, Traveling waves of dissipative non-autonomous hyperbolic equations in a strip, Adv. Differential Equations 3, 249-270 (1998)

[21] V. Pata and A. Zucchi, Attractors for a damped hyperbolic equation with linear memory, submitted

[22] R. Temam, Infinite-dimensional dynamical systems in mechanics and physics, Springer-Verlag, New York, 1988 